\title{
Les enseignements de 7 années de plan de surveillance des oléagineux
}

\author{
Sylvie DAUGUET ${ }^{1}$ \\ Florence LACOSTE ${ }^{2}$ \\ ${ }^{1}$ CETIOM, \\ Centre technique interprofessionnel \\ des oléagineux et du chanvre, \\ 11 rue Monge, \\ parc industriel \\ 33600 Pessac, \\ France \\ $<$ dauguet@cetiom.fr $>$ \\ 2 ITERG, \\ Institut des Corps Gras, \\ 11 rue Monge, \\ parc industriel \\ 33600 Pessac, \\ France \\ $<$ f.lacoste@iterg.com $>$
}

Article reçu le 13 septembre 2012

Accepté le 24 septembre 2012

La filière oléagineuse française $s^{\prime}$ est mobilisée autour de la sécurité sanitaire depuis le début des années 2000 qui correspondent à la mise en place d'un ensemble de réglementations européennes dénommées Paquet Hygiène (Dauguet et al., 2006). C'est dans ce contexte que le Plan de Surveillance de la qualité sanitaire des Oléagineux (PSO) a été mis en place de façon opérationnelle dès la campagne 2005, contribuant à contrôler la qualité des produits (graines, tourteaux et huiles) dans un cadre interprofessionnel. Depuis le lancement du PSO, de plus en plus d'opérateurs de la filière oléagineuse en sont devenus des partenaires actifs. Cet article propose un bilan sur ces sept années de fonctionnement du PSO.

\begin{abstract}
Lessons from seven years of oilseeds survey plan
Oilseeds food chain operators are coordinated through a food safety survey plan, in order to get a realistic picture of the contamination in oilseed products (seeds, oilseed meal, and vegetable oil). Concerned crops are those cultivated or processed in France: rapeseed, sunflower and soybean. Grain storage companies, feeding industries and oil industries participate voluntarily, and send their self-data that are pooled in a database. The food safety of oilseeds survey plan allows to identify which are main concerns, for instance post-harvest insecticide residues from cross contamination during storage. Results of this monitoring plan were transmitted to the French government and the European Commission in cases of revisions of regulatory thresholds.
\end{abstract}

Key words: oilseeds, vegetable oil, survey plan, contaminants, pesticide residues

Aujourd'hui, chaque opérateur de la chaîne alimentaire est confronté à l'obligation légale: (1) de mettre en place une démarche HACCP ${ }^{1}$, basée sur une analyse pertinente des risques sanitaires inhérents à son activité, (2) d'assurer la conformité sanitaire des produits qu'il met sur le marché, (3) de disposer d'un plan d'autocontrôle.

Le Plan de Surveillance des Oléagineux, mis en place par le CETIOM, I'ITERG et I'ONIDOL $^{2}$ depuis 2005 est un observatoire de la qualité sanitaire des oléagineux produits en France. Ce plan repose sur une base mutualisée de données d'analyses de contaminants sur oléagineux, qui est privée. Cette base est alimentée par les données d'autocontrôle des industriels et des organismes stockeurs (OS) qui adhèrent à ce plan, ainsi que par les campagnes d'analyses sanitaires ciblées menées sur

\footnotetext{
${ }^{1}$ HACCP : Hazard Analysis Critical Control Point, méthode et principes de gestion de la sécurité sanitaire des aliments.

${ }^{2}$ ONIDOL : Organisation nationale interprofessionnelle des oléagineux.
}

graines, tourteaux et huiles par le CETIOM, I'ONIDOL et I'ITERG (figure 1).

Destiné aux organismes stockeurs, aux transformateurs industriels de I'huilerie et aux fabricants d'aliment du bétail $(F A B)$, il concerne :

- les graines oléagineuses suivantes: colza, tournesol, soja ;

- les produits suivants : graines, tourteaux, huiles brutes et raffinées, coproduits du raffinage ;

- les contaminants suivants : résidus de produits phytopharmaceutiques, éléments traces métalliques, mycotoxines, toxiques organiques, salmonelles, impuretés botaniques, etc.

La confidentialité des données est garantie pour les partenaires, et aucune exploitation commerciale de cette base n'est faite. La base de données sur graines et tourteaux est gérée par le CETIOM, et la base de données sur huiles brutes et huiles raffinées est gérée par I'ITERG.

Le PSO est donc un outil au service de la filière oléagineuse, permettant une défense collective sur les aspects

Pour citer cet article : Dauguet S, Lacoste F. Les enseignements de 7 années de plan de surveillance des oléagineux. OCL 2013; 20(2) : 119-123. doi : 10.1684/ocl.2012.0476 


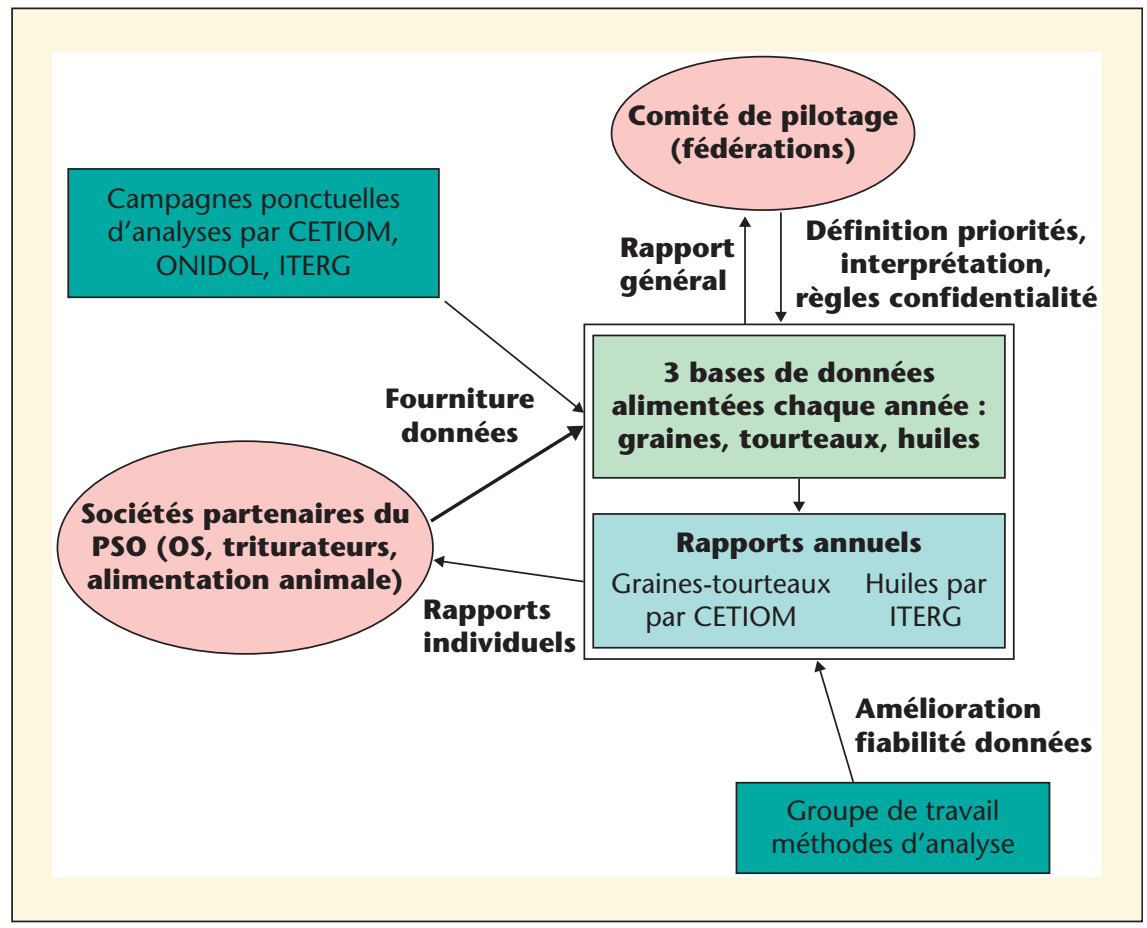

Figure 1. Fonctionnement du PSO (Plan de surveillance de la qualité sanitaire des oléagineux).

sanitaires, mettant en évidence les progrès réalisés et contribuant à la fixation de seuils réglementaires réalistes. C'est aussi un lieu d'échange entre les opérateurs de la filière, où sont identifiées des pistes de recherche pertinentes.

\section{Une bonne représentativité}

À ce jour, le PSO compte 29 partenaires actifs : 24 OS répartis sur toute la France qui représentent 30-40 \% de la collecte de graines oléagineuses en France, quatre industriels de I'huilerie (les principaux groupes présents en France), et un partenaire de la fabrication d'aliments pour animaux, I'association OQUALIM, qui fédère 57 entreprises de ce secteur (plus de $71 \%$ de la production d'aliment du bétail). La représentativité des partenaires du PSO est donc correcte par rapport à l'ensemble des opérateurs de la filière oléagineuse française. Ce plan est ouvert à toutes autres entreprises intéressées et de nouveaux adhérents le rejoignent chaque année.

Ainsi, chaque partenaire fournit les données d'analyses issues de son propre plan d'autocontrôle (laboratoire d'ana- (hydrocarbures aromatiques polycycliques, dioxines et $P(B)$, contaminants microbiologiques (salmonelles dans les tourteaux), impuretés botaniques (cas des graines de Datura spp. dans les graines de tournesol), les huiles minérales ou des composés susceptibles de se former lors du raffinage tels que les esters de 3-MCPD et les esters de glycidol dans I'huile.

\section{Fiabilité des données du PSO ?}

Les résultats d'analyses de la base de données du PSO sont issus d'une vingtaine de laboratoires, chaque partenaire du PSO pouvant choisir librement le laboratoire effectuant les analyses sur ses produits. Afin de contribuer à la fiabilisation des données, un travail collectif a été mené par I'ITERG et le CETIOM concernant l'analyse de résidus de pesticides dans les graines oléagineuses et dans I'huile car il n'existe pas de méthode normalisée spécifique pour ces matrices et que cet exercice est reconnu comme étant particulièrement difficile (complexité de cette matrice, difficultés de purification pour extraction des résidus de pesticides de la matière grasse). Ainsi, ce travail collectif a réuni environ 25 laboratoires, et a permis la rédaction d'une méthode commune qui a fait l'objet d'essais circulaires. Cependant, les résultats de ces essais circulaires n'étant pas assez bons, cette méthode n'a pas pu être proposée à la normalisation. Ce travail qui s'est déroulé sur la période 2004-2007 a cependant permis aux laboratoires français de globalement améliorer leurs pratiques et partager les meilleures techniques pour l'analyse de résidus de pesticides dans les matrices oléagineuses. Plus récemment, I'envoi par les coordinateurs du PSO d'échantillons d'huile brute dopée à une douzaine de laboratoires qui contribuent à la base de données du plan a permis de constater que l'analyse de résidus de pesticides dans une telle matrice est toujours délicate à réaliser, puisque rares ont été les laboratoires ayant des résultats jugés excellents. Une molécule telle que la deltaméthrine apparaît en particulier difficile à doser à des faibles teneurs, car elle a été fréquemment non détectée ou sous-quantifiée par les laboratoires participants. Suite à l'analyse des échantillons dopés, des échanges avec ces laboratoires ont eu lieu afin de permettre une 


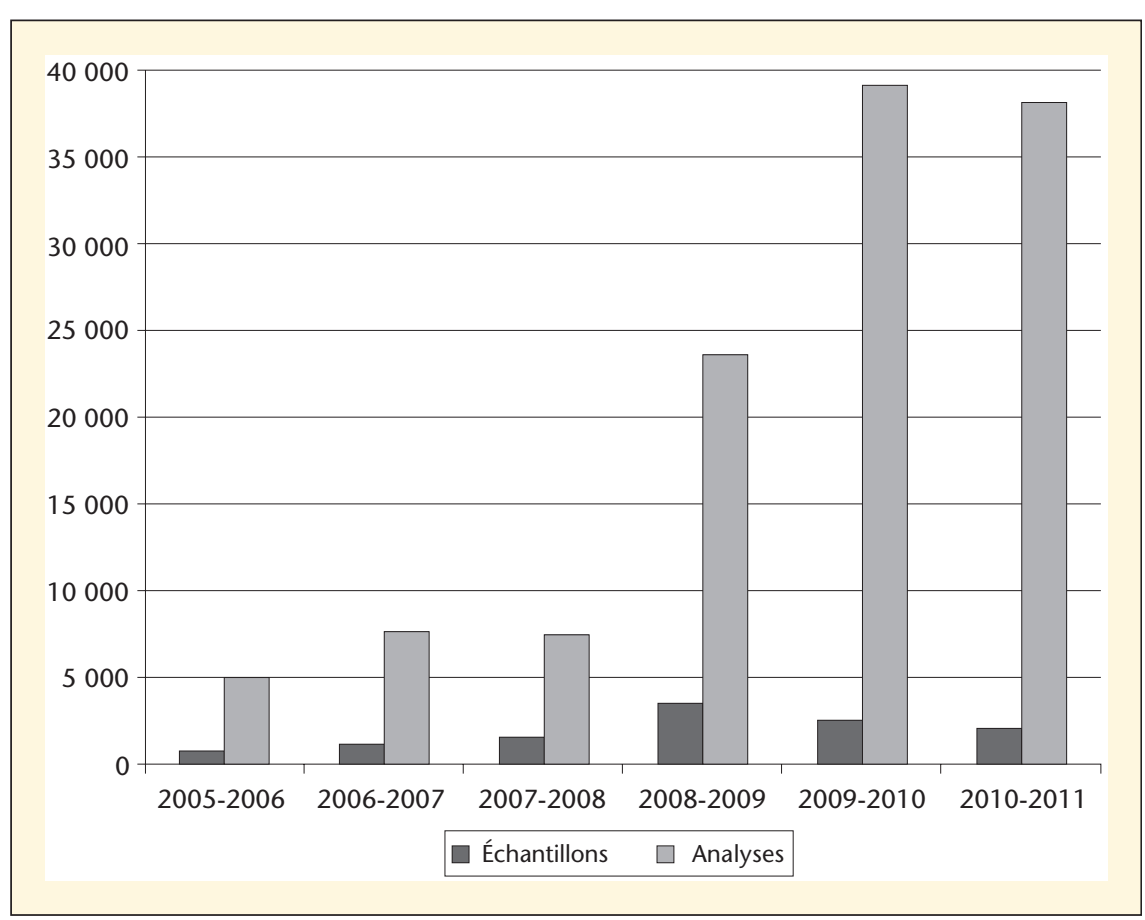

Figure 2. Evolution du nombre annuel d'échantillons et du nombre annuel de résultats d'analyse intégrés dans la base de données du PSO (Plan de surveillance de la qualité sanitaire des oléagineux).

démarche d'amélioration concernant ce type d'analyse.

\section{Les résultats du PSO}

Le PSO nous permet de vérifier que la quasi-totalité des produits oléagineux sont conformes à la réglementation en vigueur. Les seuils réglementaires concernant les oléagineux sont définis dans différents textes : limites maximales de résidus de pesticides (LMR, Règlement CE $n^{\circ} 396 / 2005$ et règlements le modifiant), teneurs maximales applicables en alimentation animale (Directive 2002/32/CE et textes la modifiant), teneurs maximales dans les denrées alimentaires pour I'alimentation humaine (Règlement $n^{\circ} 1881$ / 2006 et autres règlements le modifiant).

Cependant, le PSO donne le constat que le raffinage des huiles est nécessaire pour éliminer certains résidus de produits phytopharmaceutiques des huiles brutes. II s'agit principalement d'insecticides utilisés en post-récolte (pyrimiphos-méthyl, chlorpyriphos-méthyl, deltaméthrine), appliqués sur les locaux de stockage vides et/ou sur les céréales stockées dans les mêmes sites, se retrou- de poursuivre une surveillance de cette famille de toxines car un seuil réglementaire en aflatoxines pour I'alimentation humaine a été récemment fixé pour les graines destinées à une consommation humaine directe $(2 \mu \mathrm{g} /$ $\mathrm{kg}$ pour l'aflatoxine B1 et $4 \mu \mathrm{g} / \mathrm{kg}$ pour les aflatoxines totales), sans transformation industrielle (exemple du tournesol de bouche), avec des teneurs maximales bien plus basses qu'en alimentation animale $(20 \mu \mathrm{g} / \mathrm{kg}$ pour l'aflatoxine B1) et qu'il est possible d'atteindre très ponctuellement dans la production française de tournesol. Concernant $d^{\prime}$ autres toxines non encore réglementées telles que les toxines d'Alternaria, $I^{\prime} E F S A^{3}$ recommande aux États membres d'acquérir des données sur les teneurs présentes dans les aliments. Dans le cadre du PSO, des analyses sur ces toxines ont été réalisées et leur présence peut être occasionnellement constatée sur tournesol. Cependant les études toxicologiques ne sont pas suffisamment étayées à ce jour pour conclure sur le risque représenté par les toxines d'Alternaria.

Les éléments traces métalliques ne constituent pas une famille de contaminants à risque pour les oléagineux puisqu'ils n'occasionnent pas de dépassements de seuils réglementaires, même si dans le cas du cadmium, les teneurs retrouvées dans les graines et tourteaux de tournesol peuvent parfois se rapprocher du seuil fixé en alimentation animale $(1 \mathrm{mg} / \mathrm{kg})$.

Un contaminant a été identifié plus récemment dans le cadre du PSO : il s'agit des graines de Datura spp., qui sont des impuretés botaniques que l'on peut retrouver dans les récoltes de graines de tournesol, dans le cas de parcelles envahies par cette " mauvaise herbe ". Cette plante est toxique, car contenant dans toutes ses parties des alcaloïdes tropaniques, et la présence de graines de Datura spp. est réglementée dans les matières premières de l'alimentation animale $(1000 \mathrm{mg} / \mathrm{kg}$ de graines entières de Datura). En effet, les alcaloïdes contenus dans ces impuretés vont se retrouver, suite au procédé de trituration des graines de tournesol, préférentiellement dans les tourteaux. Des teneurs en graines de Datura dépassant parfois le seuil réglementaire

${ }^{3}$ EFSA : European Food Safety Authority. 
ont pu être observées lors de la récolte 2011 de tournesol, dues à des conditions de culture qui ont favorisé des levées tardives de cette plante.

Les substances toxiques organiques, telles que les hydrocarbures aromatiques polycycliques (HAP) et les dioxines et $P C B$, sont plus particulièrement surveillées dans les huiles brutes et raffinées. Les teneurs mesurées pour ces substances dans le cadre du PSO montrent que ces substances ne posent pas de problème particulier dans la filière oléagineuse française.

Récemment, la présence d'esters de 3MCPD et d'esters de glycidol, composés néoformés au cours du raffinage dans certaines conditions, a été signalée dans des huiles végétales raffinées ainsi que dans des produits alimentaires formulés contenant des matières grasses végétales (Zelinkova, 2006). L'huile de palme est I'huile présentant les taux de contamination les plus élevés vraisemblablement en lien avec les températures élevées de désodorisation utilisées lors du raffinage physique, alors que les huiles de graines sont globalement moins sujettes à la formation de ce contaminant (Kuhlmann, 2011). Les quelques résultats collectés via le PSO confirment la faible contamination des huiles raffinées de colza et tournesol.

À la suite d'une contamination d'huiles brutes de tournesol en provenance d'Ukraine par des huiles minérales (Lacoste, 2010), les industriels ont mis en place depuis 2008 une vérification systématique des huiles de tournesol d'importation. Les données collectées dans le cadre du PSO permettent de vérifier que cette contamination de 2008 était un cas isolé.

\section{PSO, un outil au service de la filière oléagineuse}

Les résultats du PSO permettent donc aux opérateurs de la filière de mener une analyse sur les dangers sanitaires dans les produits oléagineux. Ainsi, le sujet des résidus $d^{\prime}$ insecticides post-récolte est apparu important et mobilisateur, ce qui a poussé les professionnels de la filière à mener des actions spécifiques pour identifier les sources de contaminations des graines oléagineuses par ces résidus dans les installations de stockage. Les enquêtes menées en collaboration avec les entreprises du secteur

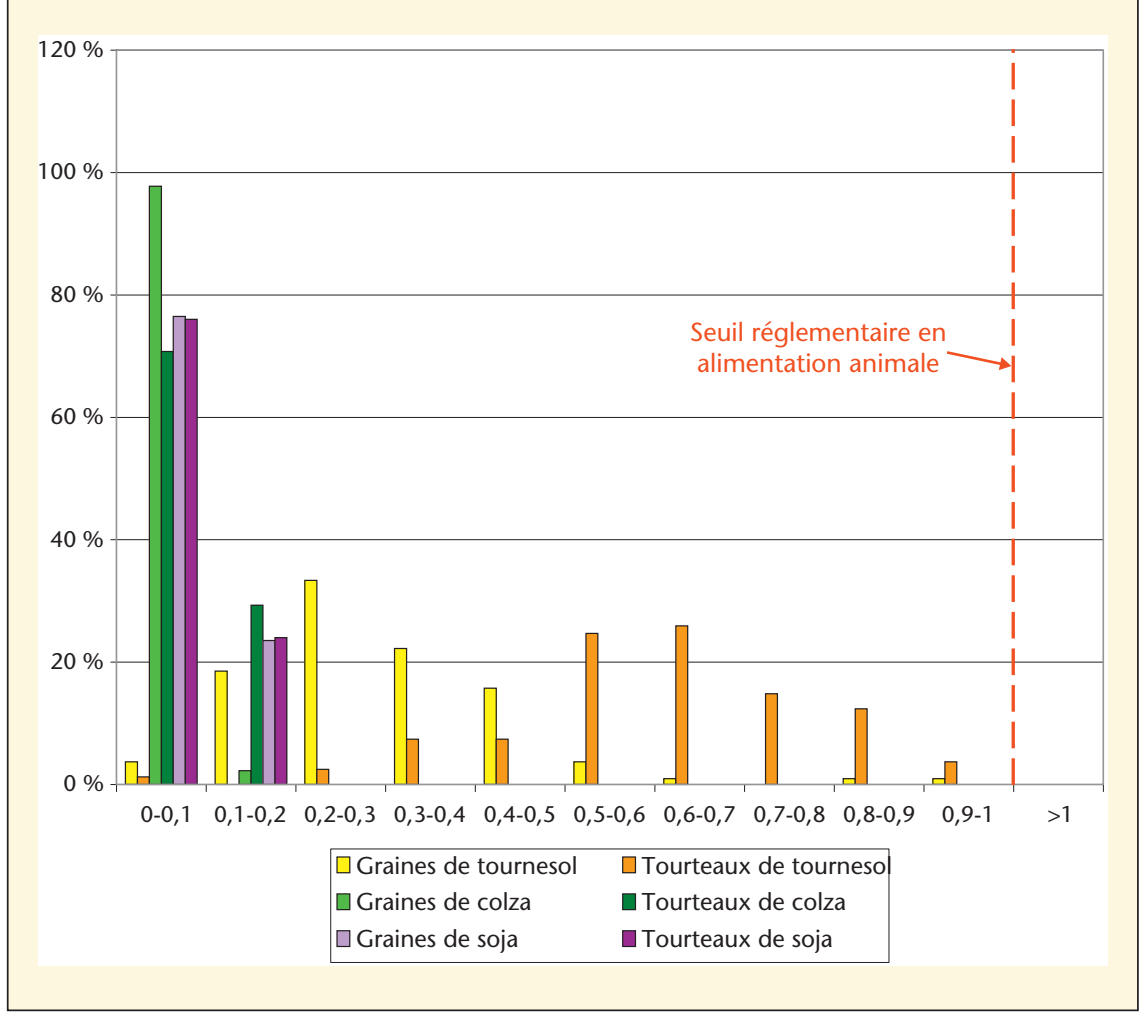

Figure 3. Répartition des échantillons par classes de teneurs en cadmium ( $\mathrm{mg} / \mathrm{kg}$ ) dans les graines et tourteaux d'oléagineux (données PSO 2003-2009).

ont permis l'identification des situations menant à ces contaminations croisées, ce qui a fait l'objet de publications (Dauguet, 2007 ; Dauguet, 2009), et de recommandations relayées par les fédérations des organismes de collectestockage. Selon les derniers résultats du PSO, les teneurs en ces résidus ont tendance à baisser, ce qui montre une prise en compte de cette problématique par les professionnels grâce à la communication qui a été faite autour du sujet. Le PSO a également été mis à contribution dans le cadre d'un dossier remis à la Commission européenne par la FEDIOL $^{4}$ en juin 2011 afin d'argumenter pour l'augmentation de la limite réglementaire du pyrimiphos-méthyl pour les graines oléagineuses, prenant en compte le phénomène de contamination croisée durant le stockage. Ce dossier, ainsi que des dossiers remis par les autres professions concernées (COCERAL, EUROMAISIERS FERM et EFM), ont été étudiés par I'EFSA qui a publié une opinion (EFSA, 2011) dans

\footnotetext{
${ }^{4}$ FEDIOL : Fédération européenne des industries de I'huile végétale et des tourteaux.
}

laquelle l'autorité de sécurité sanitaire européenne indique qu'une LMR de $0,5 \mathrm{mg} / \mathrm{kg}$ serait appropriée notamment sur graines oléagineuses (alors que la LMR actuelle est de $0,05 \mathrm{mg} / \mathrm{kg}$ ). Par la suite, les résultats du PSO concernant les résidus en chlorpyriphosméthyl (autre insecticide post-récolte) sur graines de colza et tournesol ont également été mis à contribution dans un courrier adressé par FEDIOL à la Commission européenne en janvier 2012 pour la prise en compte par la réglementation du phénomène de contamination croisée au stockage par cette substance. Le Plan de Surveillance des Oléagineux, tel qu'il est mené en France, présente donc un réel intérêt pour la filière oléagineuse afin $d$ 'apporter des éléments objectifs d'argumentation dans le cadre de révision des réglementations.

Dans le cadre de la révision des seuils réglementaires en cadmium dans I'alimentation humaine, les partenaires du PSO se sont également mobilisés pour apporter aux pouvoirs publics des données afin que les futurs seuils ne soient pas une entrave au commerce 
des graines oléagineuses. Ce sujet concerne surtout le tournesol, qui accumule du cadmium dans ses graines. Aujourd'hui, aucun dépassement du seuil réglementaire de l'alimentation animale en vigueur n'est observé pour le tournesol, mais un seuil plus bas pourrait poser problème (figure 3 ). II semblerait que la future réglementation ne portera que sur les graines destinées à une consommation directe, et donc ne s'appliquerait pas aux graines destinées à la transformation en huile et tourteau. Concrètement, des données du PSO ont été transmises aux autorités françaises dans le cadre des discussions européennes sur la révision des seuils, après accord de l'ensemble des membres du comité de pilotage du PSO. Cela illustre également l'intérêt de disposer de données fiables afin de contribuer à la fixation de seuils réglementaires réalistes.

\section{Les perspectives d'évolution du PSO}

Ce Plan de Surveillance est considéré aujourd'hui comme une action pérenne, pour le bénéfice des opérateurs de la filière oléagineuse française, qui n'a pas d'équivalent dans les autres pays. Cela implique des moyens humains pour le faire vivre au niveau du CETIOM et de I'ITERG. A ce jour, des réflexions sont en cours pour améliorer le fonctionnement du PSO en utilisant plus efficacement les nouveaux moyens de communication et d'information. L'idée serait notamment d'apporter plus de réactivité et de souplesse en introduisant une saisie en ligne des données et une consultation des résultats globaux facilitée par un site internet dédié et protégé. Ces évolutions ont bien sûr un coût qu'il sera intéressant de partager avec d'autres filières ayant les mêmes besoins, tels que la filière céréalière qui dispose d'un Plan de surveillance sanitaire des céréales (PSSC) piloté par I'IRTAC.

Les entreprises (organismes stockeurs, industries de l'huilerie et fabricants d'aliment du bétail) qui sont partenaires du PSO expriment I'intérêt qu'elles portent à cette démarche et leur soutien par leur implication, montrant ainsi qu'une action collective au niveau $d^{\prime}$ une filière de production est possible. Les résultats du PSO sont privés et n'ont pas vocation à être publiquement diffusés, mais seulement avec I'accord de I'ensemble des partenaires du PSO pour des besoins ciblés pour la défense des intérêts de la filière.

Ce type de mutualisation de données intéresse à plus large échelle les pouvoirs publics français, y voyant la possibilité d'objectiver le niveau de sécurité sanitaire des aliments en dehors des temps de crise et de valoriser les activités de surveillance privées et publiques: il s'agit du volet sanitaire de l'Observa- toire de I'alimentation, qui est en cours de construction, sous la responsabilité de la DGAL et mis en œuvre par I'ANSES.

\section{Conflits d'intérêts : aucun}

\section{RÉFÉRENCES}

Dauguet S, Lacoste F, Ticot B, Loison JP, Evrard I, Bouchtane B, Soulet B. La filière oléagineuse se mobiliser autour de la problématique des résidus d'insecticides. $O C L$ 2006 ; 6 : 373-7.

Dauguet S. Insecticide residues cross-contamination of oilseeds during storage. $O C L$ 2007 ; 6 : 313-6.

Dauguet $S$. Insecticide residues cross-contamination of oilseeds during storage (second part). OCL 2009 ; 3 : 144-8.

EFSA. Risk assessment for pirimiphos-methyl residues resulting from cross-contamination. EFSA Journal $2011 ; 11$ : 2436-83.

Kuhlmann J. Determination of Bound 2,3Epoxy-1-propanol (Glycidol) and Bound Monochloropropanediol (MCPD) in Refined Oils. Eur J Lipid Sci Technol 2011 ; 113 : 335-44.

Lacoste $F$, Joffre F, Coustille JL, Morin O, Soulet B, Brenne E, Griffon H. Détection de contaminants dans les huiles végétales : bilan à fin 2009. OCL $2010 ; 2$ : 75-80.

Zelinkova Z, Svejkovska J, Velisek J, Dolezal M. Fatty acid esters of 3-chloropropane-1,2diol in edible oils. Food additives and contaminants $2006 ; 23: 1290-8$. 